\title{
Editorial overview: Memory in time and space
}

\author{
Lila Davachi ${ }^{1}$ and Neil Burgess ${ }^{2}$
}

1. Psychology, Columbia University NY 10027
e-mail: Id24@columbia.edu

2. Institute of Cognitive Neuroscience, University College London, United Kingdom e-mail:n.burgess@ucl.ac.uk

Our memory for the events we have personally experienced forms a crucial part of our consciousness, helps to define how we think about ourselves and guides our future decisions. Psychologists and neuroscientists have studied the nature and representation of these 'episodic' memories for decades and, while much progress has been made, there is still much to learn. Being able to 're-experience,' or at least reinstate, the spatiotemporal context of the event, that is where and when it occurred, has long been held to be a defining characteristic of episodic memory [1]. Equally, the hippocampus has long been identified as a key player in episodic memory [2]. While it is clear that hippocampal loss leads to an inability to remember not just spatial and temporal aspects of memory but also all manner of relational and/or associative aspects of memory [3], the mechanisms supporting episodic memory still remain elusive.

In this issue we bring together reviews of some of the recent theoretical and experimental advances in understanding the nature of the representations of content and context that underlie memory, the brain systems that support them and their influences on the way we remember or forget various aspects of the stream of experience.

A breakthrough into the neural representations present within the hippocampal formation came with the discovery of neurons in freely moving rodents whose firing directly reflected their environmental location ('place cells,' [4]) or head-direction [5], followed more than thirty years later by the discovery of grid cells [6] and boundary-coding cells $[7,8]$. Early work showed that the hippocampus was necessary for tasks requiring internal representation of environmental locations in rodents [9] and in humans (see e.g. [10] for a review). More recently, neural firing in these regions in rodents has been shown to reflect the passage of time with in a task ('time cells,' [11]), and indeed other continuous task-relevant parameters of experience [12].

Here, Poucet and Hok review evidence concerning how neuronal processing in hippocampal and frontal areas enable rodents to remember, and return to, the spatial location of a reward. In humans, Ekstrom and Isham review how spatial navigational strategies vary flexibly with the scale and dimensionality of the task in hand. Computational accounts of how the spatial and temporal properties of neuronal firing in and around the hippocampus could arise from attractor dynamics and network oscillations, and underpin memory function, are reviewed by Hasselmo et al.

As seen in the earlier work on spatial memory, cross-species and developmental approaches can provide powerful insights. Rueckemann and Buffalo consider hippocampal 'view' cells in primates that respond not when an animal navigates through a particular location but rather when it looks at it (see also [13]), and use viewing behaviour to argue that hippocampal cells respond not just to places but also to other aspects of ongoing experience. Jelbert and Clayton examine the relationship between memory abilities in corvids and humans using a developmental approach, while Ghetti 
shows that different kinds of memory associations have different developmental trajectories. Work in mouse models allows the use of powerful opto-genetic and chemo-genetic manipulations which can reveal how mnemonic representations are allocated, consolidated and integrated, as reviewed by Schlichting and Frankland. These techniques also enable direct visualization and manipulation of the 'engram' cells encoding a specific memory, as reviewed by Tonegawa and Roy.

Recent neuropsychological evidence that the MTL is required for remembering the durations and temporal orders of events is reviewed by Palombo and Verfaellie. In terms of neural mechanisms, Teki et al. describe a computational model of how oscillatory dynamics could explain the behavioural data concerning our perception and memory for the passage of time. These oscillatory dynamics have intriguing similarity to those used in models of spatial coding ([14]; Hasselmo et al.), and are reflected in the temporal organisation of place and grid cell firing relative to the theta rhythm ('theta phase precession' $[15,16])$. At the population level theta phase precession is seen as sequential patterns of activity sweeping from representing places behind the animal to those in front [17-19]. Here, Zutshi et al.argue that theta sweeps across grid cells can provide a movement direction signal to the rest of the brain. Wang and Diana review the evidence for distinct contributions of frontal and medial temporal lobe regions to temporal memory consistent with other dissociations highlighting the unique contributions of MTL cortex and hippocampus to other aspects of episodic memory $[25,26]$, while Robin and Moscovitch discuss how the long-term passage of time influences the nature of individual episodic memories.

Several reviews offer the synthesis that memory for spatial and temporal relations are examples of a broader relational memory function $[27,28,29]$. Manohar et al. review elegant recent experiments showing how even short-term memory for such information requires the hippocampal formation, while Rondina and Ryan discuss the association of spatial and temporal processing with different frequencies of oscillatory activity, and argue that temporal relations are the foundation for other aspects of memory. Consistent with a widespread focus on the predictive brain across fields spanning perception to memory [30,31], Gravina and Sederberg present a view of perception and memory as predictive, so that both time and space are measures of relevance derived from features. Gershman shows how viewing memory in terms of its ability to predict the future can explain a broad range of phenomena from temporal contiguity effects to the firing patterns of place cells.

Recently, work on memory has highlighted the idea that integration across memories is a critical function of our memory systems [32,33]; Following on this idea, Collin et al. discuss how both spatial and mnemonic representations can be thought of as networks of interlinked elements, stored in a hierarchical system allowing the integration of new information. Morton et al. review experiments concerning how new events are integrated with existing knowledge in hippocampus and medial prefrontal cortex, and how this allows novel generalization. Finally, linking relational memory to temporal processing, Eichenbaum describes how time cells could implement a time-varying context to which specific individual elements of experience can become associated to provide memory for temporally organised experiences.

Many authors have investigated the nature of the representation of 'context' in which events are embedded from theoretical grounds, with much work following the framework of the 'Temporal Context Model' (TCM) for the inter-dependence of experience, context and memory retrieval [20]. Here, Howard reviews recent theoretical advances in formal models of spatiotemporal context, and evidence that neuronal populations implement these models. From a complementary experimental perspective, Polyn and Cutler review recent experiments testing the predictions following from different ways of representing temporal context, while Cohn-Sheehy and Ranganath highlight the 
role of neural representations in the hippocampus and posterior medial brain network that promote the temporal organization of experiences.

One important aspect of temporal context is that it is dependent on our experiences, both internal and external, and those experiences or thoughts have the power to reset or retime the context signal and have been shown to have consequences on how are memories are organized $[34,35,36]$. Exactly how this discretization or chunking occurs is of crucial importance to memory research. Here, Radvansky and Zacks review current thinking on 'event' or 'situation' models of how this happens, with a focus on discontinuous changes of context, whether spatial (e.g. going through a doorway) or semantic (e.g. a switch in category of stimuli) in nature. Following this line of thinking, DuBrow et al. review recent evidence for signs that memory formation corresponds to discontinuous shifts in the neural representation of context. Clewett and Davachi review emerging work showing that sequential integration within events and separation at event boundaries are supported by distinct neural and behavioral signatures.

Recent work on 'episodic future thinking' [21] emphasizes the similarities between episodic simulation and memory. Schacter et al. review the continuing success of this idea and highlight how episodic simulation contributes to other cognitive domains and discuss the functions of contributing brain areas. A core aspect of episodic simulation involves the construction and/or imagery of coherent spatial scenes which goes back to ideas about the role of place and head direction cells in imposing a single viewpoint onto the retrieval of environmental information [22,23], providing perhaps an explanation for the role of the hippocampus in imagery [24] and suggesting roles for other regions such as retrosplenial cortex. Here, Dalton and Maguire review recent evidence for a specific role for pre-subiculum and para-subiculum in holistic scene representations. This view of the distinct associative mechanisms supporting coherent imagery versus those allowing memory for individual items is reviewed by Bisby and Burgess in terms of the differential effects of encoding traumatic information on the two systems, and their implications for post-traumatic stress disorder.

Overall we hope that this issue serves to outline some of the many current theoretical and experimental approaches to understanding the neural systems, mechanisms and representations supporting memory. The sophistication and proliferation of these approaches, and the common threads of spatial, temporal, contextual and integrative representations promise a rapidly increasing understanding of this crucial cognitive function in the coming years.

\section{References}

1. Tulving E: Elements of Episodic Memory. Clarendon; 1983.

2. Scoville WB, Milner B: Loss of recent memory after bilateral hippocampal lesions. J Neurol Neurosurg Psychiatry 1957, 20:11-21.

3. Cohen NJ, Eichenbaum H: Memory, Amnesia, and the Hippocampal System. MIT Press; 1993.

4. O'Keefe J, Dostrovsky J: The hippocampus as a spatial map. Preliminary evidence from unit activity in the freely-moving rat. Brain Res 1971, 34:171-175.

5. Taube JS, Muller RU, Ranck JB: Head-direction cells recorded from the postsubiculum in freely moving rats. I. Description and quantitative analysis. J Neurosci 1990, 10: 420-435.

6. Hafting T, Fyhn M, Molden S, Moser MB, Moser El: Microstructure of a spatial map in the entorhinal cortex. Nature 2005, 436:801-806. 
7. Solstad T, Boccara CN, Kropff E, Moser MB, Moser El: Representation of geometric borders in the entorhinal cortex. Science 2008, 322:1865-1868.

8. Lever C, Burton S, Jeewajee A, O'Keefe J, Burgess N: Boundary vector cells in the subiculum of the hippocampal formation. J Neurosci 2009, 29:9771-9777.

9. Morris RGM, Garrud P, Rawlins JNP, O'Keefe J: Place navigation impaired in rats with hippocampal lesions. Nature 1982, 297:681-683.

10. Burgess N, Maguire EA, O'Keefe J: The human hippocampus and spatial and episodic memory. Neuron 2002, 35:625-641.

11. McDonald CJ, Lepage KQ, Eden UT, Eichenbaum H: Hippocampal "time cells" bridge the gap in memory for discontiguous events. Neuron 2011, 71:737-749.

12. Aronov D, Nevers R, Tank DW: Mapping of a non-spatial dimension by the hippocampalentorhinal circuit. Nature 2017, 543:719-722.

13. Georges-Francois P, Rolls ET, Robertson RG: Spatial view cells in the primate hippocampus: allocentric view not head direction or eye position or place. Cereb Cortex 1999, 9: 197-212.

14. Burgess N, Barry C, O'Keefe J: An oscillatory interference model of grid cell firing. Hippocampus 2007, 17:801-812.

15. O'Keefe J, Recce ML: Phase relationship between hippocampal place units and the EEG theta rhythm. Hippocampus 1993, 3:317-330.

16. Hafting T: Hippocampus-independent phase precession in entorhinal grid cells. 2008.

17. Burgess N, Recce M, O’Keefe J: A model of hippocampal function. Neural Netw 1994, 7:10651081.

18. Skaggs WE, McNaughton BL, Wilson MA, Barnes CA: Theta phase precession in hippocampal neuronal populations and the compression of temporal sequences. Hippocampus 1996, 6:149-172.

19. Johnson A, Redish AD: Neural ensembles in CA3 transiently encode paths forward of the animal at a decision point. J Neurosci 2007, 27:12176-12189.

20. Howard MW, Kahana MJ: A distributed representation of temporal context. J Math Psychol 2002, 46:269-299.

21. Addis DR, Wong AT, Schacter DL: Remembering the past and imagining the future: common and distinct neural substrates during event construction and elaboration. Neuropsychologia 2007, 45:1363-1367.

22. Byrne $\mathrm{P}$, Becker $\mathrm{S}$, Burgess $\mathrm{N}$ : Remembering the past and imagining the future: a neural model of spatial memory and imagery. Psychol Rev 2007, 114:340-375.

23. Burgess N, Becker S, King JA, O'Keefe J: Memory for events and their spatial context: models and experiments. Philos Trans R Soc Lond B Biol Sci 2001, 356:1493-1503.

24. Hassabis D, Kumaran D, Vann SD, Maguire EA: Patients with hippocampal amnesia cannot imagine new experiences. Proc Natl Acad Sci U S A 2007, 104:1726-1731.

25. Davachi L: Item, Context and Relational Episodic encoding in humans. Curr. Opin. Neurol 2006, 16(6):693-700.

26. Eichenbaum $\mathrm{H}$, Yonelinas AP, Ranganat $\mathrm{C}$ : The medial temporal lobe and recognition memory. Annu Rev Neurosci 2007, 30: 123-152. 
27. Cohen NJ, Eichenbaum H: Memory, amnesia, and the hippocampal system. Cambridge, Mass: MIT Press; 1993.

28. Eichenbaum $\mathrm{H}$, Cohen $\mathrm{NJ}$ : From conditioning to conscious recollection memory systems of the brain. Oxford: Oxford University Press; 2001.

29. Eichenbaum $\mathrm{H}$, Cohen NJ: Can we reconcile the declarative memory and spatial navigation views on hippocampal function? Neuron 2014, 83(4):764-770.

30. Trapp S, Bar M: Prediction, context and competition in visual recognition. Ann N Y Acad Sci. 2015, 1339:190-198.

31. Davachi L, DuBrow S: How the hippocampus preserves order: the role of prediction and context. Trends Cogn Sci 2015, 19(2):92-99. 32. Shohamy D, Wagner AD: Integrating memories in the human brain: hippocampal-midbrain encoding of overlapping events. Neuron 2008, 60(2):378-389.

33. Schlichting ML, Preston AR: Memory integration: neural mechanisms and implications for behavior. Curr Opin Behav Sci 2015, 1:1-8.

34. Ezzyat Y, Davachi L: What constitutes an episode in episodic memory? Psychol Sci 2011, 22(2):243-252.

35. Ezzyat $Y$, Davachi L: Similarity breeds proximity: pattern similarity within and across contexts is related to later mnemonic judgments of temporal proximity. Neuron 2014, 81(5):1179-1189.

36. DuBrow S, Davachi L: The influence of context boundaries on memory for the sequential order of events. J Exp Psychol Gen 2013, 142(4):1277-1286. 